\title{
Recent advances in superconducting rotating machines: an introduction to the 'Focus on Superconducting Rotating Machines'
}

\author{
Mark Ainslie ${ }^{1}$, Mitsuru Izumi², Motohiro Miki ${ }^{2}$ \\ ${ }^{1}$ Bulk Superconductivity Group, Department of Engineering, University of Cambridge, \\ Trumpington Street, Cambridge CB2 1PZ, UK \\ ${ }^{2}$ Laboratory of Applied Physics, Tokyo University of Marine Science and Technology, 2-1-6 \\ Etchujima, Koto-ku, Tokyo 135-8533, Japan
}

Superconductivity offers zero to near zero resistance to the flow of electrical current when cooled below a particular cryogenic temperature, and additionally, superconducting materials are able to carry much larger current densities than conventional materials, such as copper. In rotating machines, increasing the current and/or magnetic flux density increases the power density, which leads to reductions in both size and weight of the machine. The expected improved performance and efficiency, as well as smaller footprint, has seen continued interest in using superconducting materials in rotating machine designs. In this preface, we introduce some of the recent advances in superconducting rotating machines and their related technologies that form this special issue.

Over several decades of research, various superconducting machines have been shown to be technically feasible over a wide range of power ranges, for applications in electricity generation (conventional, hydroelectric, and wind turbines) and motors for ship and aircraft propulsion and electric vehicles. For the first such attempts - synchronous generators using low temperature superconducting (LTS) materials - the complexity and cost of $4 \mathrm{~K}$ cryogenics hindered the commercial development of these machines [1], although there were a number of successful technical feasibility demonstrations [2]. The discovery of high temperature superconducting (HTS) materials renewed enthusiasm for applied superconductivity research with the expectation that these materials could be exploited at $77 \mathrm{~K}$, the boiling point of liquid nitrogen. Since then, a number of projects around the world have demonstrated the technical feasibility of HTS machines in various forms. Many of these demonstrators have been based on the first generation (1G) HTS conductors (BSCCO) [3-6]; however, the focus has recently shifted towards the second generation (2G) HTS conductors ((RE)BCO, where (RE) is a rare earth element or yttrium), which exhibit comparatively better superconducting properties.

In [7], Moon et al report the design, fabrication and preliminary tests of a megawatt-class 2G HTS motor, designed specifically for ship propulsion, at Doosan Heavy Industries and Construction. The rotor consists of racetrack coils wound using GdBCO wire, which is cooled by forced circulation of liquid neon to achieve an operating temperature less than $30 \mathrm{~K}$. The preliminary tests showed measured values consistent with the design specification, but a 
number of technical issues, such as coil impregnation, improving mechanical strength and reducing the necessary cooling time, are raised as future considerations.

The stator in this case is based on conventional technology, and until now, many other designs have focused chiefly on this kind of design: an isolated, cryogenic rotor and conventional stator, or in other words, a hybrid superconducting machine based on conventional synchronous machine design. However, a conductor with low AC losses would allow for the use of HTS conductors in both the stator and rotor windings, leading to the development of an all-cryogenic or all-superconducting machine with unprecedented power densities. In [8], Song et al provide a comprehensive analysis of the thermal behaviour of an HTS stator employing BSCCO windings on an iron core, cooled to $82.1 \mathrm{~K}$ by liquid nitrogen, and a permanent magnet (PM) rotor.

Another technical challenge for superconducting rotating machines with a rotating DC field winding is the need for brushes/slip-rings as a rotating joint. In [9], Bumby et al demonstrate a prototype brushless HTS-PM exciter to avoid this problem, making use of a flux pump to inject current into a closed HTS coil without the need for a direct electrical connection to the rotor, which additionally removes thermal penalties associated with current leads. The exciter can also operate externally across a cryostat wall without forming a thermal bridge.

In order to avoid rotating magnets/coils and AC losses, Fuger et al have developed superconducting homopolar machines, which utilise only DC electric and magnetic fields for operation. The operating principle and design of such machines is presented in [10], including the first experimental results from a $200 \mathrm{~kW}$ motor demonstrator. Liquid metal current collectors deliver a low resistance, stable and low maintenance sliding contact to the rotor. In [11], Radyjowski et al report on an initial prototype claw-pole superconducting machine that has a modular design, as well as a stationary superconducting field winding, eliminating the need for cryocouplers, brushes and so on. Campbell analyses the possibility and performance of a superconducting magnetic gear using HTS conductors in [12], which could replace a conventional gearbox in an electric machine and offer reduced acoustic noise and vibration, as well as improved reliability and significantly reduced maintenance.

There is great interest in the application of superconducting machines in direct drive wind turbines for wind power generation, particularly for the offshore market, which demands reliable, lightweight and high power density solutions. Additionally, there is considerable interest in applications of $\mathrm{MgB}_{2}$ superconducting materials as an alternative to LTS and HTS materials, offering a number of advantages as low cost and density conductor with potentially low AC losses. In [13], Marino et al report the design of a $10 \mathrm{MW}, 8.1 \mathrm{rpm}$ superconducting generator for direct drive, offshore wind turbines that uses $\mathrm{MgB}_{2}$ field coils cooled to $20 \mathrm{~K}$ and a conventional, ambient temperature stator winding. A weight reduction of $26 \%$ in comparison to a conventional PM generator is estimated, which would allow a tower $11 \%$ lighter. As part of the same SUPRAPOWER project, Vargas-Llanos et al use finite element analysis in [14] to estimate the hysteretic losses in multi-filamentary $\mathrm{MgB}_{2}$ tapes 
under the operating conditions of the SUPRAPOWER prototype generator, including an AC transport current and a DC transport current under an external applied magnetic field, with and without ripples from the coupling between the stator and rotor. The influence of filament transpositions within the tapes on the AC loss is also examined.

Bulk superconducting materials, which acting as trapped field magnets (TFMs) can trap magnetic fields of magnitude over ten times that of conventional permanent magnets [15], also have the potential to act as permanent magnet analogues in superconducting rotating machines. The TUMSAT group in Japan has worked on axial gap-type superconducting rotating machines employing bulk HTS materials for over a decade and [16] provides an overview of their developments to date, including bulk material fabrication, pulsed field magnetisation and experimental test results on a number of prototype machines. Finally, the design and build of a fully superconducting magnetic bearing (SMB) system using bulk YBCO and Nd-Fe-B permanent magnets is reported by $\mathrm{Xu}$ et al in [17]. This has potential application in high speed rotating machines as a superior, friction-free bearing with low loss and noise.

\section{References}

[1] Tixador P 2010 Physica C 470 971-979

[2] Shimada T et al 1999 IEEE Trans. Appl. Supercond. 9 1189-1192

[3] Nick W, Grundmann J and Frauenhofer J 2012 Physica C 482 105-110

[4] Snitchler G, Gamble B and Kalsi S S 2005 IEEE Trans. Appl. Supercond. 15 2206-2209

[5] Gamble B, Snitchler G and MacDonald T 2011 IEEE Trans. Appl. Supercond. 21 1083-1088

[6] Fair R, Lewis C, Eugene R and Ingles M 2010 J. Phys.: Conf. Ser. 234032008

[7] Moon H, Kim Y-C, Park H-J, Yu I-K and Park M 2016 Supercond. Sci. Technol. 29034009

[8] Song P, Qu T-M, Lai L-F, Wu M-S, Yu X-Y and Han Z 2016 Supercond. Sci. Technol. 29 054007

[9] Bumby C W et al 2016 Supercond. Sci. Technol. 29024008

[10] Fuger R, Matsekh A, Kells J, Sercombe D B T and Guina A 2016 Supercond. Sci. Technol. 29034001

[11] Radyjowski P, Keysan O, Burchell J and Mueller M 2016 Supercond. Sci. Technol. 29 044002

[12] Campbell A 2016 Supercond. Sci. Technol. 29054008 
[13] Marino I et al 2016 Supercond. Sci. Technol. 29024005

[14] Vargas-Llanos C R, Zermeno V M R, Sanz S, Trillaud F and Grilli F 2016 Supercond. Sci. Technol. 29034008

[15] Durrell J H et al 2014 Supercond. Sci. Technol. 27082001

[16] Zhang Y, Zhou D, Ida T, Miki M and Izumi M 2016 Supercond. Sci. Technol. 29044005

[17] Xu K-X, Wu D-J, Jiao Y L and Zheng M H 2016 Supercond. Sci. Technol. 29064001 